\title{
Mechanisms of hippocampal sequence replay
}

\author{
Paola Malerba*, Giri P Krishnan, Maxim Bazhenov \\ From 24th Annual Computational Neuroscience Meeting: CNS*2015 \\ Prague, Czech Republic. 18-23 July 2015
}

Sleep is known to be important for memory consolidation [1], and memories are thought to be stored in the hippocampus during wakefulness and "transferred" to cortex during sleep [2]. Recently, memory replay repeatable sequences of pyramidal cell firing - has been demonstrated during sleep, and associated with characteristic brain oscillations, giving rise to the hypothesis that these may form the critical neural substrate of memory consolidation. It is known that the content of hippocampal replay can be biased during sleep, in what is called cued-reactivation [3], and that similar paradigms show enhanced memory performance in humans [4]. Moreover, tampering with replay can disrupt memory formation and consolidation [5]. Despite extensive evidence highlighting the importance of replay within the broader phenomenon of sleep-mediated memory consolidation, the mechanisms underlying sequence replay are still unknown.

During sleep, replay events are associated with specific patterns of neuronal oscillations [6]. Replay is seen in cortex during slow oscillation - a rhythmic $(<1 \mathrm{~Hz})$ state in which periods of activity (active or Up states) alternate with quiet periods (silent or Down states), while replay in the hippocampus is associated with sharp-wave ripple events - irregularly brief bouts of high frequency $(>150 \mathrm{~Hz})$ firing, driven by strong excitatory inputs coming from CA3, which result in a strong deflection in the LFP of stratum radiatum (the sharp wave) [7].

In the present study, we build on our previous research [8] to develop a model of spike sequence replay during sleep. In the past, we have introduced a model of CA1 ripples in which oscillations are transients, mediated by the intrinsic frequency of CA1 basket cells driven by CA3 activation. In this work, we construct a model of CA3 area in which stochastic intrinsic activation of pyramidal cells originates a massive cell activation that results in a

\footnotetext{
* Correspondence: paola.malerba@ucr.edu

Cell Biology and Neuroscience, University of California Riverside, Riverside,
} CA 92507, USA strong excitatory input to area CA1. We observe that sequential activation of a selected sub-group of CA1 pyramidal cells is driven by a less specific sequential activation in CA3. We then characterize the mechanisms underlying sequence selection and reactivation within a sharp-wave ripple event.

Using hippocampal and thalamocortical models, we investigate the role of cortical inputs on ripple timing and their specific spike content. Our study illustrates the possible role of cortical Up states during slow oscillation in biasing hippocampal replay, which is a core component of the cortico-hippocampal interaction underlying memory consolidation.

\section{Acknowledgements}

Published: 18 December 2015

\section{References}

Rasch B, Born J: About sleep's role in memory. Physiol Rev 2013, 93(2):681-766.

. Marr D: Simple memory: a theory for archicortex. Philos Trans $R$ Soc Lond B Biol Sci 1971, 262(841):23-81.

3. Bendor D, Wilson MA: Biasing the content of hippocampal replay during sleep. Nat Neurosci 2012, 15(10):1439-1444.

4. Cairney SA, Durrant SJ, Hulleman J, Lewis PA: Targeted memory reactivation during slow wave sleep facilitates emotional memory consolidation. Sleep 2014, 37(4):701-707, 707A.

5. Nakashiba T, Buhl DL, McHugh TJ, Tonegawa S: Hippocampal CA3 output is crucial for ripple-associated reactivation and consolidation of memory. Neuron 2009, 62(6):781-787.

6. Ji D, Wilson MA: Coordinated memory replay in the visual cortex and hippocampus during sleep. Nat Neurosci 2007, 10(1):100-107.

7. Ylinen A, Bragin A, Nadasdy Z, Jando G, Szabo I, Sik A, Buzsaki G: Sharp wave-associated high-frequency oscillation $(200 \mathrm{~Hz})$ in the intact hippocampus: network and intracellular mechanisms. J Neurosci 1995, 15(1 Pt 1):30-46.

8. Bazhenov M, Timofeev I, Steriade M, Sejnowski TJ: Model of thalamocortical slow-wave sleep oscillations and transitions to activated States. J Neurosci 2002, 22(19):8691-8704.

\section{doi:10.1186/1471-2202-16-S1-P11}

Cite this article as: Malerba et al:: Mechanisms of hippocampa sequence replay. BMC Neuroscience 2015 16(Suppl 1):P11. 